\title{
AS DIMENSÕES DO EMPREENDEDORISMO INTERNACIONAL: UMA PROPOSIÇÃO DE UM FRAMEWORK
}

\section{RESUMO}

Este ensaio teórico tem o objetivo de apresentar um framework de empreendedorismo internacional (EI). A proposta está fundamentada na revisão crítica de pesquisas empíricas e teóricas internacionais e nacionais dessa área promissora. Os textos analisados foram selecionados nas principais bases científicas disponíveis no Brasil, a saber: Periódicos CAPES, Proquest e Ebsco. Como resultado da pesquisa, seis dimensões do empreendedorismo foram identificadas, quais sejam: propensão à adaptação, redes de relacionamentos, oportunidades internacionais, capacidade de inovar, atitude face ao risco e desenvolvimento de recursos competitivos. Tais elementos são expressados em eventos marcantes da internacionalização empresarial que compõem as fases com incidência intermitente ao longo da trajetória da internacionalização. Além disso, como implicação para investigações futuras, observa-se que os fatores de processo e contextuais favorecem a aplicação do framework de EI.

Palavras-chave: Empreendedorismo Internacional; Negócio Internacional; Empreendedorismo.

\section{THE DIMENSIONS OF INTERNATIONAL ENTREPRENEURSHIP: A PROPOSITION OF A FRAMEWORK}

\begin{abstract}
This theoretical paper aims to present a framework of international entrepreneurship (IE). The proposal is based on the critical review of theoretical and empirical international and national researches of this promising area. The texts analyzed were selected in major scientific bases available in Brazil, namely: CAPES Journals, Proquest and Ebsco. As result of the research, six dimensions of entrepreneurship were identified, namely: propensity to the adaption, social networks, international opportunities, ability to innovate, attitude towards risk and the development of competitive resources. Theses elements are expressed in key events of business internationalization with intermittent incidence along the trajectory of internationalization. Moreover, as implications for future research, it is observed that the process and contextual factors favor the application of the EI framework .
\end{abstract}

Keywords: International Entrepreneurship; International Business; Entrepreneurship. 


\section{LAS DIMENSIONES DE LA INICIATIVA EMPRESARIAL INTERNACIONAL: UNA PROPUESTA DE UN}

\section{MARCO}

\section{RESUMEN}

Este trabajo teórico tiene como objetivo presentar un marco de la iniciativa empresarial internacional (IE) . La propuesta se basa en la revisión crítica de las investigaciones internacionales y nacionales teóricos y empíricos de este sector prometedor. Los textos analizados fueron seleccionados en las principales bases científicas disponibles en Brasil, a saber: CAPES Revistas, Proquest y Ebsco. Como resultado de la investigación, se identificaron seis dimensiones de la iniciativa empresarial, a saber: el ajuste de tendencia, las redes sociales, las oportunidades internacionales, capacidad de innovación, la actitud hacia el riesgo y el desarrollo de recursos competitivos. Tales elementos se expresan en los acontecimientos clave de la internacionalización de las empresas con incidencia intermitente a lo largo de la trayectoria de internacionalización. Por otra parte, como las implicaciones para futuras investigaciones, se observa que el proceso y los factores contextuales favorecen la aplicación del marco de la IE.

Palabras-clave: La Iniciativa Empresarial Internacional, Negocios Internacionales; La Iniciativa Empresarial.

\footnotetext{
${ }^{1}$ Doutora em Administração pela Universidade Federal de Pernambuco - UFPE. Professora da Universidade Federal Rural do Semi-Árido - UFERSA. Brasil. E-mail: yakarav@gmail.com

${ }^{2}$ Doutor em Mangament Sciences pela University of Manchester Institute of Science and Technology, Grã-Bretanha. Professor da Universidade Federal de Pernambuco - UFPE. Brasil. E-mail: walter.moraes@ufpe.br
} 


\section{INTRODUÇÃO}

O empreendedorismo internacional (EI) é uma temática contemporânea. Nos EUA, os estudos de McDougall e Oviatt, iniciados no final da década de 1980, tornaram-se referência na área (McDougall, 1989; OViatt \& McDougall, 1994; Oviatt \& McDougall, 2005a; Oviatt \& McDougall, 2005b; Oviatt \& McDougall, 2005c).

No Brasil, com base na consulta realizada no banco de teses e dissertações da Coordenação de Aperfeiçoamento de Pessoal de Nivel Superior (CAPES), em dezembro de 2011, verificou-se que apenas duas dissertações (Freitag Filho, 2008; Rossi, 2008) e três teses (Dib, 2008; Galimberti, 2009; Mello, 2009) tratam direta ou indiretamente do tema. Foram localizados onze artigos, nos eventos da Associação Nacional de Pós-Graduação e Pesquisa em Administração (ANPAD) e em periódicos nacionais. Esse fato está alinhado ao que é apresentado por Butler et al. (2010), quando explicam que os estudos de empreendedorismo internacional se concentram nos EUA, por isso, defendem a necessidade da realização de pesquisas sobre o EI em outros países.

Atualmente, observa-se que o empreendedorismo internacional é um fenômeno presente nas empresas novas, antigas, pequenas e grandes, porém, a maioria dos pesquisadores ainda continua a centralizar as pesquisas nas firmas jovens e pequenas (Coombs et al., 2009). O que, de alguma maneira, dificulta descobertas capazes de ajudar nos avanços da área. Nessa linha, este trabalho tenta contribuir ao selecionar empresas maduras e de maior porte na tentativa de identificar sutilezas do fenômeno.

Há outros aspectos em torno do EI passíveis de discussão, a questão de como e por que algumas empresas se internacionalizam cedo e rapidamente é uma delas (Keupp \& Gassmann, 2009). A tecnologia é identificada como uma das principais forças propulsoras da aceleração da internacionalização (Oviatt \& McDougall, 2005a). Por outro lado, diferentemente do mainstream, Welch e Welch (2004) elaboraram uma proposta teórica com dados empíricos de um caso do agronegócio, que não é comum por ser o EI tradicionalmente estudado nas empresas pequenas do setor de tecnologia. Nesse contexto, não foi essencialmente a tecnologia determinante, mas sim as redes de relacionamentos, as ações empreendedoras e as políticas tiveram papel fundamental para a internacionalização. Esse resultado expõe como o EI nas empresas do agronegócio é expressado e como a continuação de estudos nesse segmento poderá trazer revelações significativas ao campo de pesquisa, assim, foi também nesse sentido que se buscou colaborar.

$\mathrm{Na}$ literatura revisada, existem outras limitações, seja por tratar o contexto do EI apenas como cultura organizacional (Dimitratos \& Plakoyannaki, 2003), por considerar no modelo teórico de EI diferentes tipos de estratégias sem incluir as estratégias internacionais (Zahra \& George, 2002) e por ser rara a realização de estudos longitudinais (Coombs et al., 2009). Tal método é mais adequado para a identificação de ações empreendedoras na internacionalização ao longo do tempo (Keupp \& Gassmann, 2009), sendo este o procedimento metodológico considerado nesta pesquisa para cobrir esse espaço.

Ademais, alguns pontos podem ser explorados. Este ensaio teórico tem o objetivo geral de apresentar um framework de empreendedorismo internacional. A revisão bibliográfica permite identificar três pilares que apoiam a elaboração do objetivo, são eles: i) influência do contexto no empreendedorismo internacional; ii) inexistência de um framework que considere as características das empresas brasileiras; e iii) propensão à adaptação, redes de relacionamentos, oportunidades internacionais, capacidade de inovar, atitude face ao risco e desenvolvimento de recursos competitivos são evidenciados na literatura, mas, não compõem de forma concomitante os modelos teóricos de EI.

Sendo assim, nenhum dos frameworks revisados apresenta simultaneamente as seis dimensões típicas de EI, identificadas na literatura especializada, numa concepção contextual e da internacionalização como um processo. O que faz desse fato o terceiro pilar na composição do objetivo desta investigação.

Quanto à estrutura, além desta introdução, o ensaio teórico está organizado em mais três seções. A seguir, discute-se sobre empreendedorismo internacional. Depois, é apresentado o framework de EI, para na sequência, expor as considerações finais. Finalmente, as referências são apresentadas.

\section{REFERENCIAL TEÓRICO}

\subsection{Empreendedorismo Internacional}

O estado da arte do empreendedorismo internacional destaca que Morrow (1988) foi o primeiro a escrever sobre o tema. Todavia, McDougall (1989) é considerada pioneira por realizar a primeira pesquisa teórico-empírica (Acs et al. 2003).

A temática vem sendo discutida em edições especiais de periódicos internacionais e fóruns direcionados ao EI, desde 1996, havendo demonstrações do crescente interesse acadêmico (Jones et al., 2011; Oviatt \& McDougall, 2005a). Além disso, o Handbook of Research on International Entrepreneurship (Dana, 2004) e o Journal of International Entrepreneurship, periódico internacional especializado em EI, são duas publicações relevantes na área, que devem ser mencionadas diante de sua contribuição acadêmica.

Em 2009, o estudo do empreendedorismo internacional completou duas décadas. Fato que 
motivou a elaboração de estudos bibliográficos que sumarizaram e avaliaram o estado da arte dessa corrente teórica (Coombs et al., 2009; Jones et al., 2011; Keupp \& Gassmann, 2009; Kiss et al., 2012) e da metodologia de EI (Coombs et al., 2009; Coviello \& Jones, 2004; Keupp \& Gassmann, 2009).

O EI possui algumas características que o distingue do empreendedorismo tradicional. $\mathrm{O}$ empreendedorismo está relacionado à criação de um novo negócio por parte do empreendedor (Filion, 1999). Esse entendimento também é compartilhado pelo Global Entrepreneurship Monitor (Kelley et al., 2010). Mas, no empreendedorismo internacional a questão não está apenas na criação de negócios, o aspecto central consiste na forma em que ocorre o processo de internacionalização. $\mathrm{O}$ empreendedorismo internacional está presente nas firmas que se internacionalizam por meio de ações empreendedoras que podem ou não ser implementadas pelo proprietário (Dimitratos \& Plakoyiannaki, 2003).

Para aprofundar a discussão, realiza-se um exercício de reflexão com base nos artigos internacionais revisados. Alguns construtos se destacam, a saber: atitude face ao risco, inovação, internacionalização, oportunidades internacionais, processo, recursos competitivos e vantagem competitiva.

No empreendedorismo internacional, o risco está no investimento realizado para atender o mercado internacional, na negociação com os compradores que muitas vezes não tem garantias de pagamento (McDougall, 1989) e no modo de entrada no exterior (Welch \& Welch, 2004). Embora, tenha sido contemplada apenas na conceituação de Mtigwe (2006), a sua discussão é considerada por diferentes autores que serão apresentados ao longo do desenvolvimento deste capítulo. A atitude face ao risco, além de ser um fator preponderante na literatura clássica de empreendedorismo, vem ganhando espaço nos estudos de internacionalização. Tajeddini e Mueller (2009) argumentam que o comportamento de enfrentar os riscos faz parte das características do empreendedor, sendo uma das dimensões mais relevantes.

A inovação é também primordial, trata-se de um dos fatores que caracteriza o EI como área do conhecimento. Permite a identificação de oportunidades internacionais, delimita a configuração da cadeia de suprimentos, seleciona áreas a serem internacionalizadas, define caminhos únicos para passar valor ao consumidor e contribui na identificação de oportunidades (Mtigwe, 2006; Zahra; George, 2002). Essa proposta mais ampla se diferencia do que Oviatt e McDougall (2005a) defendem, por limitar a inovação aos produtos e serviços comercializados internacionalmente.

A internacionalização é enfatizada em algumas das conceituações, o que está em completa conformidade com a proposta teórica do empreendedorismo internacional. As atividades empresariais se voltam ao exterior (Coombs; SADRIEH; ANNAVARJULA, 2009) e podem iniciar o negócio, motivadas por essa razão (McDougall, 1989).

O processo de exploração de oportunidades internacionais é um dos elementos-chave do empreendedorismo internacional. Dimitratos e Plakoyiannaki (2003) reconhecem o EI como sendo um processo que ocorre em determinado contexto, aproximando-se do entendimento de Mtigwe (2006). Em relação às oportunidades no mercado internacional, Zahra e George (2002) defendem que a sua exploração pode ser influenciada pela criatividade empreendedora de vários membros do time gerencial. Enquanto que para Dimitratos e Plakoyiannaki (2003), a identificação de oportunidades é insuficiente, a habilidade de explorá-las é essencial, argumento semelhante ao defendido por Oviatt e McDougall (2005a).

A questão processual do empreendedorismo internacional é percebida por autores contemporâneos. Há pesquisadores que entendem o EI como um processo em si (Coombs; SADRIEH; ANNAVARJULA, 2009), podendo também ser motivado pela busca para conquistar a criação de valor (Mtigwe, 2006) e descobrir oportunidades (Zahra; George, 2002). É um fenômeno que tende a ser melhor observado em um período mais longo de tempo, devido ao seu caráter processual.

Os recursos competitivos apoiam a internacionalização (Oviatt; McDougall, 1994) para se alcançar a vantagem competitiva. Os recursos não apenas precisam ser heterogêneos e imóveis, mas também, valiosos, raros, insubstituíveis e inimitáveis elementos do modelo VRII ou ter organização para explorá-los, constituindo o modelo VRIO (Barney, 2002). Nesse sentido, o capital financeiro, o físico, o humano e o organizacional são categorias de recursos. Quanto às capacidades, são reconhecidas como atributos internos da empresa que permitem a coordenação e exploração dos recursos, enquanto que as competências favorecem à implementação das estratégias (Barney, 2002).

A partir da revisão de literatura, alguns construtos se destacam, a saber: a exploração de oportunidades internacionais (Dimitratos \& Plakoyiannaki, 2003; Mtigwe, 2006; Oviatt \& McDougall, 2005a; Zahra \& George, 2002), a inovação (Mtigwe, 2006; Zahra \& George, 2002), a atitude face ao risco (Mtigwe, 2006), o desenvolvimento de recursos competitivos (Oviatt \& McDougall, 1994) e a vantagem competitiva (Oviatt \& McDougall, 1994; Zahra \& George, 2002). Portanto, o empreendedorismo internacional é resultante do processo de exploração de oportunidades internacionais, da inovação, da atitude face ao risco e do desenvolvimento de recursos competitivos por parte do empreendedor para obter vantagem competitiva. Tal compreensão conceitual revela-se robusta por estar embasada nos principais fatores observados nos conceitos de EI apresentados 
por Coombs, Sadrieh e Annavarjula (2009), Dimitratos e Plakoyiannaki (2003), McDougall (1989), Mtigwe (2006), Oviatt e McDougall (1994), Oviatt e McDougall (2005a) e Zahra e George (2002). A partir dessa definição e do framework pretende-se contribuir no desenvolvimento da ciência proporcionando um modelo teórico capaz de oferecer a articulação de elementos essenciais do fenômeno.

\subsection{Panorama dos Estudos de EI Publicados no Brasil}

Empreendedorismo, empreendedorismo internacional, internacionalização, entrepreneurship, international entrepreneurship, international business e international foram as palavras-chave utilizadas durante a pesquisa bibliográfica de EI no Brasil. No total, 113 revistas acadêmicas, com melhor avaliação na área de Administração indicadas na CAPES, com Qualis A1, A2, B1 e B2, anais dos encontros da Associação Nacional de Pós-Graduação e Pesquisa em Administração - ANPAD (incluindo o EnAnpad 2011), teses e dissertações cadastradas no banco de dados da CAPES e currículos lattes $(\mathrm{CNPq})$ dos pesquisadores brasileiros foram examinados na internet.

Identificaram-se dois artigos nos periódicos analisados, o primeiro na Revista de Administração Mackenzie (Honório, 2008) e o segundo nos Cadernos EBAPE.BR (Ferreira et al., 2010). A motivação para se internacionalizar é o tema principal da pesquisa quantitativa de Honório (2008) desenvolvida com informações coletadas em 73 empresas brasileiras exportadoras de manufaturas. A literatura de EI é contemplada superficialmente para examinar a influência do empreendedor como um dos motivadores da internacionalização. No ensaio teórico de Ferreira $e t$ al. (2010), os pesquisadores discutem a participação das redes no contexto das pequenas e médias empresas e propõem uma tipologia que é produto dessa relação.

Em três eventos organizados pela ANPAD, nove artigos foram localizados, que de modo direto e indireto retratam o empreendedorismo internacional ou o papel do empreendedor na internacionalização. Salienta-se que no caso do EnAnpad, realizou-se a busca nas edições disponibilizadas no site da associação (de 1997 a 2011) e no CD dos anais de 2012, totalizando seis artigos, dentre eles, dois teóricos
(Child \& Rodrigues, 2007; Dib \& Carneiro, 2006) e quatro teórico-empíricos (Freitag Filho \& Amal, 2008; Ribeiro \& Pimentel, 2009; Sohn et al., 2004; Tondolo et al., 2008). No 3Es, um dos papers é teórico (Mello et al., 2009) e outro, teórico-empírico (Silveira \& Alperstedt, 2007). Enquanto que no Simpósio apenas um trabalho teórico foi publicado (Galimberti \& Fracasso, 2008).

No banco de teses e dissertações da CAPES, localizaram-se duas dissertações de mestrado. Rossi (2008) identifica e analisa as dimensões teóricas do empreendedorismo internacional no processo de internacionalização de uma grande empresa mineira do setor alimentício. A literatura de EI é discutida em conjunto com a de redes de relacionamentos na dissertação de mestrado (Freitag Filho, 2008). Há também três teses de doutorado. As born globals fazem parte do tema principal do trabalho de Dib (2008), com isso, o EI é apresentado transversalmente. Do mesmo modo, Galimberti (2009) adota a perspectiva do EI para dar suporte à pesquisa de pequenas e médias empresas de softwares, enquanto que no trabalho de Mello (2009), são apresentadas as contribuições do EI em conjunto com mais três perspectivas teóricas de internacionalização (modelo de Uppsala, a teoria de redes e os estudos sobre born globals).

Desse modo, são onze artigos apresentados nas revistas e eventos mais relevantes do campo de conhecimento das ciências administrativas, duas pesquisas de mestrado e três de doutorado publicadas, totalizando 16 trabalhos. O primeiro foi divulgado depois de 15 anos da publicação do artigo seminal de EI (McDougall, 1989). Sohn et al. (2004) estudaram a influência do espírito empreendedor na internacionalização de uma empresa de software, sem adotar a perspectiva teórica de empreendedorismo internacional. Todavia, foi apenas com Dib e Carneiro (2006) que o EI foi discutido a partir dos autores clássicos da área.

A figura 1 mostra o resultado da análise das publicações brasileiras, e revela os principais temas abordados nessas pesquisas. Ressalta-se ainda que a pesquisa bibliográfica aconteceu no período entre janeiro de 2011 a março de 2012. 
As Dimensões do Empreendedorismo Internacional: Uma Proposição de um Framework

\begin{tabular}{|c|c|}
\hline TEMAS & DESCRIÇÃ $O$ \\
\hline $\begin{array}{l}\text { Aprendizagem e } \\
\text { conhecimento }\end{array}$ & $\begin{array}{l}\text { - conhecimento dos nichos de mercado internacionais (Galimberti \& Fracasso, 2008) } \\
\text { - conhecimento e aprendizagem por meio da internacionalização incremental (Rossi, 2008; Silveira } \\
\text { \& Alperstedt, 2007; Sohn, Lenzi \& Kiesel, 2004) } \\
\text { - experiência anterior do processo de internacionalização, para entrada em novos mercados } \\
\text { (Tondolo, Bitencourt \& Tondolo, 2008) } \\
\text { - para obter recursos (Tondolo, Bitencourt \& Tondolo, 2008) } \\
\text { - conhecimentos tecnológicos e científicos específicos (Ribeiro \& Pimentel, 2009) }\end{array}$ \\
\hline Barreiras & $\begin{array}{l}\text { - burocracia e limitação financeira (Freitag Filho \& Amal, 2008) } \\
\text { - falta de recursos (Freitag Filho \& Amal, 2008) } \\
\text { - idioma do país anfitrião (Ribeiro \& Pimentel, 2009) }\end{array}$ \\
\hline Confiança & $\begin{array}{l}\text { - redes (Fernandes \& Seifert Júnior, 2007; Mello, Rocha \& Maculan, 2009), parcerias e } \\
\text { distribuidores (Child \& Rodrigues, 2007) } \\
\text { - colabora na diminuição da distância psíquica (cultura e diferenças institucionais) (Child \& } \\
\text { Rodrigues, 2007) } \\
\text { - os laços podem ser emocionais, ultrapassando os tradicionais e institucionais (Child \& Rodrigues, } \\
\text { 2007) } \\
\text { - depende de aspectos pessoais, como sentimento e intuição (Child \& Rodrigues, 2007) }\end{array}$ \\
\hline Empreendedor & $\begin{array}{l}\text { - interesses e crenças (Fernandes \& Seifert Júnior, 2007; Honório, 2008) } \\
\text { - visão (Dib, 2008; Dib \& Carneiro, 2006; Ribeiro \& Pimentel, 2009; Silveira \& Alperstedt, 2007; } \\
\text { Sohn, Lenzi \& Kiesel, 2004) } \\
\text { - motivação (Dib \& Carneiro, 2006; Honório, 2008) } \\
\text { - determinação (Silveira \& Alperstedt, 2007) } \\
\text { - centralizção de poder de decisão (Sohn, Lenzi \& Kiesel, 2004) } \\
\text { - vontade de abrir novos horizontes de mercado (Fernandes \& Seifert Júnior, 2007; Sohn, Lenzi \& } \\
\text { Kiesel, 2004) } \\
\text { - tomador de decisão (Dib; Carneiro, 2006; Galimberti \& Fracasso, 2008) } \\
\text { - gestão de recursos (Tondolo, Bitencourt \& Tondolo, 2008) } \\
\text { - promove a capacitação do RH (Ribeiro \& Pimentel, 2009) } \\
\text { - desenvolvem e fortalecem redes (Mello, 2009; Mello, Rocha \& Maculan, 2009) } \\
\text { - perpassa toda a empresa, envolvendo não somente o presidente ou proprietário (Rossi, 2008) }\end{array}$ \\
\hline Indústria & $\begin{array}{l}\text { - manufatura (diversas) (Honório, 2008) } \\
\text { - vinícola - agronegócio (Tondolo, Bitencourt \& Tondolo, 2008) } \\
\text { - software (Galimberti \& Fracasso, 2008; Ribeiro \& Pimentel, 2009; Sohn, Lenzi \& Kiesel, 2004) } \\
\text { - indústria alimentícia (Freitag Filho, 2008; Rossi, 2008) } \\
\text { - moveleira (Silveira \& Alperstedt, 2007) } \\
\text { - indústria de plásticos; pescado; calçados; eletrônicos (Freitag Filho, 2008) }\end{array}$ \\
\hline Inovação & $\begin{array}{l}\text { - faz parte do EI (Freitag Filho \& Amal, 2008) } \\
\text { - tecnologia - software (Galimberti \& Fracasso, 2008; Sohn, Lenzi \& Kiesel, 2004) } \\
\text { - ideias inovadoras (Fernandes \& Seifert Júnior, 2007; Silveira \& Alperstedt, 2007) } \\
\text { - criatividade (Silveira \& Alperstedt, 2007) }\end{array}$ \\
\hline $\begin{array}{l}\text { Localização das } \\
\text { empresas } \\
\text { investigadas }\end{array}$ & $\begin{array}{l}\text { - Sul (Freitag Filho \& Amal, 2008; Mello, 2009; Sohn, Lenzi \& Kiesel, 2004; Tondolo, Bitencourt } \\
\text { \& Tondolo, 2008) } \\
\text { - Sudeste (Mello, 2009; Ribeiro \& Pimentel, 2009; Rossi, 2008) } \\
\text { - Nordeste (Mello, 2009) } \\
\text { - França (Galimberti, 2009) }\end{array}$ \\
\hline Oportunidade & $\begin{array}{l}\text { - depende da capacidade do empreendedor (Ribeiro \& Pimentel, 2009; Sohn, Lenzi \& Kiesel, } \\
\text { 2004) } \\
\text { - para obter lucro e crescimento (Honório, 2008) } \\
\text { - internacionalização (Leite \& Moraes, 2012; Rossi, 2008) }\end{array}$ \\
\hline $\begin{array}{c}\text { Políticas } \\
\text { governamentais }\end{array}$ & $\begin{array}{l}\text { - apoio à internacionalização (Freitag Filho; Amal, 2008) } \\
\text { - recursos financeiros e apoio do BNDES, do FINEP, do CNPq, da FAPESP (Ribeiro; Pimentel, } \\
\text { 2009) }\end{array}$ \\
\hline $\begin{array}{l}\text { Propensão à } \\
\text { adaptação }\end{array}$ & $\begin{array}{l}\text { - flexibilidade e agilidade para responder à internacionalização (Silveira \& Alperstedt, 2007; Sohn, } \\
\text { Lenzi \& Kiesel, 2004) } \\
\text { - reestruturação (Ribeiro \& Pimentel, 2009) } \\
\text { - modificação na estrutura organizacional (Rossi, 2008) } \\
\text { - mudança no produto, na embalagem e nas campanhas publicitárias (Rossi, 2008) } \\
\text { - mudança estratégica como uma ação empreendedora (Galimberti \& Fracasso, 2008) }\end{array}$ \\
\hline Recursos & $\begin{array}{l}\text { - desenvolvidos ao longo da trajetória, tais como, as certificações (Tondolo, Bitencourt \& Tondolo, } \\
\text { 2008) } \\
\text { - elevam o padrão competitivo (Tondolo, Bitencourt \& Tondolo, 2008) } \\
\text { - marca da universidade, além de suas instalações e conhecimento científico (Ribeiro \& Pimentel, } \\
\text { 2009) }\end{array}$ \\
\hline
\end{tabular}




\begin{tabular}{|c|c|}
\hline $\begin{array}{l}\text { Redes de } \\
\text { relacionamentos }\end{array}$ & $\begin{array}{l}\text { - acesso aos recursos (Ferreira, Santos \& Serra, 2010), considerados estratégicos, tal como, } \\
\text { conhecimento de mercado (Freitag Filho, 2008; Freitag Filho \& Amal, 2008; Tondolo, Bitencourt } \\
\text { \& Tondolo, 2008) } \\
\text { - redes internacionais, regionais e locais (Ferreira, Santos \& Serra, 2010) } \\
\text { - as redes do empreendedor colaboram na identificação (Freitag Filho \& Amal, 2008; Rossi, 2008) } \\
\text { e exploração de oportunidades (Ferreira, Santos \& Serra, 2010; Mello, Rocha \& Maculan, 2009) } \\
\text { - permite o desenvolvimento de alianças estratégicas e outras formas de cooperação (Freitag Filho } \\
\text { \& Amal, 2008) } \\
\text { - favorece o desempenho (Freitag Filho \& Amal, 2008) } \\
\text { - sucesso da internacionalização (Ferreira, Santos \& Serra, 2010; Freitag Filho \& Amal, 2008) } \\
\text { - dimensões da integração do EI com redes: orientação internacional desde o início, } \\
\text { comportamento empreendedor e operações em pequena escala (Ferreira, Santos \& Serra, 2010) } \\
\text { - feiras para se atualizar e adquirir conhecimento (Ribeiro \& Pimentel, 2009; Silveira \& } \\
\text { Alperstedt, 2007) } \\
\text { - parcerias com distribuidores (Freitag Filho \& Amal, 2008; Galimberti \& Fracasso, 2008; Sohn, } \\
\text { Lenzi \& Kiesel, 2004) } \\
\text { - Intensidade do engajamento nas redes com: competidores, governos, entidades de } \\
\text { classe/empresas (Galimberti \& Fracasso, 2008) } \\
\text { - seleção (Freitag Filho \& Amal, 2008) e acesso a mercado (Ferreira, Santos \& Serra, 2010) }\end{array}$ \\
\hline Riscos & $\begin{array}{l}\text { - inevitável (Mello, Rocha \& Maculan, 2009) } \\
\text { - propensão ao risco (Fernandes \& Seifert Júnior, 2007; Freitag Filho \& Amal, 2008; Mello, Rocha } \\
\text { \& Maculan, 2009; Rossi, 2008) } \\
\text { - para ter uma internacionalização agressiva (Mello, Rocha \& Maculan, 2009) }\end{array}$ \\
\hline $\begin{array}{l}\text { Tamanho da } \\
\text { firma }\end{array}$ & $\begin{array}{l}\text { - pequenas e médias (Child \& Rodrigues, 2007; Freitag Filho \& Amal, 2008; Galimberti \& } \\
\text { Fracasso, 2008; Galimberti, 2009; Mello, Rocha \& Maculan, 2009) } \\
\text { - grandes (Dib \& Carneiro, 2006; Leite \& Moraes, 2012; Rossi, 2008) }\end{array}$ \\
\hline $\begin{array}{c}\text { Tempo de } \\
\text { funcionamento } \\
\text { da firma }\end{array}$ & $\begin{array}{l}\text { - novas (Dib, 2008; Ferreira, Santos \& Serra, 2010; Ribeiro \& Pimentel, 2009) } \\
\text { - antigas (Leite \& Moraes; 2012; Rossi, 2008; Tondolo, Bitencourt \& Tondolo, 2008) }\end{array}$ \\
\hline
\end{tabular}

Figura 1 - Temas de EI estudados no Brasil Fonte: Elaborada pelos autores.

Diante do conteúdo exposto na fiigura, algumas inferências em relação aos temas de EI podem ser apresentadas. A aprendizagem e o conhecimento são fatores que se destacam nas pesquisas desenvolvidas nas empresas brasileiras. Os empreendedores estão atentos para aprender sobre o mercado, os competidores e a gestão da cadeia de suprimentos (Rossi, 2008; Silveira \& Alperstedt, 2007; Sohn et al., 2004). O conhecimento tanto favorece a obtenção de recursos, quanto é considerado como um recurso intangível para explorar as oportunidades e reduzir os riscos (Tondolo et al., 2008).

Por outro lado, a burocracia, a limitação financeira (Freitag Filho \& Amal, 2008), a falta de recursos (Freitag Filho \& Amal, 2008) e o idioma específico do país anfitrião (Ribeiro \& Pimentel, 2009) são as principais barreiras para que as empresas brasileiras investigadas sob a ótica do EI se internacionalizem.

Em relação à confiança, impulsiona a atividade do empreendedorismo internacional. A confiança é mais importante no início da internacionalização por não se ter informações e conhecimentos suficientes, porque quando se trata de pequenas e médias empresas não se pode contar com a reputação para ter confiança. Desse modo, a sensibilidade e a intuição tendem a se sobressair sobre o aspecto racional (Child \& Rodrigues, 2007).
As ações empreendedoras são reveladas como um fator central nos estudos de EI. Elas não são exclusivas dos empreendedores conhecidos como fundadores ou proprietários. Determinados funcionários que tenham atitudes empreendedoras na internacionalização são, para o EI, empreendedores (Rossi, 2008). Esses indivíduos influenciam a internacionalização por meio dos seus interesses, crenças (Honório, 2008), visão (Dib, 2008; Ribeiro \& Pimentel, 2009; Silveira \& Alperstedt, 2007; Sohn et al., 2004), motivação (Dib \& Carneiro, 2006; Honório, 2008), determinação (Silveira \& Alperstedt, 2007), centralização de poder de decisão (Sohn et al., 2004), vontade de abrir novos horizontes de mercado (Sohn et al., 2004), nas tomadas de decisão (Dib \& Carneiro, 2006; Galimberti \& Fracasso, 2008), na gestão de recursos (Tondolo et al., 2008), por promover a capacitação do RH (Ribeiro \& Pimentel, 2009) e no desenvolvimento e fortalecimento de redes (Mello, 2009; Mello et al., 2009).

A indústria é destacada apenas para que se possa reconhecer em que mercados as empresas estudadas atuam. Percebe-se que há concentração naquelas que desenvolvem softwares (Galimberti \& Fracasso, 2008; Ribeiro \& Pimentel, 2009; Sohn et al., 2004), seguindo a tendência das pesquisas americanas na fase de surgimento do EI. Além dessas, firmas de manufatura de diversos segmentos (Honório, 2008), 
As Dimensões do Empreendedorismo Internacional: Uma Proposição de um Framework

empresas do setor de alimentos (Freitag Filho, 2008; Rossi, 2008), indústrias de plásticos, pescado, calçados, eletrônicos (Freitag Filho, 2008) e uma vinícola (Tondolo et al., 2008) foram investigadas.

Quanto à inovação, é evidenciada principalmente, por estar associada ao desenvolvimento de softwares (Galimberti \& Fracasso, 2008; Sohn et al., 2004). Todavia, criatividade, ideias (Silveira \& Alperstedt, 2007) e atitudes inovadoras fazem parte do construto do empreendedorismo, assim como do empreendedorismo internacional (Freitag Filho \& Amal, 2008).

No que se refere à localização das empresas, não se conhece a realidade do Centro-oeste e Norte do país. Foram analisadas empresas brasileiras das regiões Sul (Freitag Filho \& Amal, 2008; Sohn et al., 2004; Tondolo et al., 2008), Sudeste (Ribeiro \& Pimentel, 2009; Rossi, 2008) e Nordeste (Mello, 2009), como também, da França (Galimberti, 2009).

O empreendedor visualiza e explora oportunidades que dificilmente um indivíduo comum identificaria. Para o EI, as oportunidades estão no exterior e, para serem exploradas, é necessária a presença de capacidades empreendedoras (Ribeiro \& Pimentel, 2009; Sohn et al., 2004) para obter lucro e crescimento (Honório, 2008).

Outro fator de destaque são as políticas governamentais do país de origem, nesse caso, brasileiras. Elas são primordiais quando oferecem condições necessárias para as negociações com o estrangeiro (Freitag Filho \& Amal, 2008). Há uma multiplicidade de fontes de colaboração, o governo pode contribuir fornecendo apoio financeiro, tecnológico e tributário (Ribeiro \& Pimentel, 2009).

$\mathrm{O}$ empreendedor precisa ter a capacidade de adaptação e conduzir a empresa de modo a ter flexibilidade para atender a demanda internacional (Silveira \& Alperstedt, 2007; Sohn et al., 2004). As modificações (Ribeiro \& Pimentel, 2009) dos processos, estrutura, produto (Rossi, 2008) e até mesmo, a implementação de certificações de qualidade são essenciais para competir globalmente. Este fator não foi discutido diretamente pelos autores, apenas é apresentado de modo superficial nos resultados de algumas pesquisas.

Esses resultados mostram que o empreendedor tem responsabilidade na continuidade do negócio ao realizar a adaptação da empresa. Tal compreensão está em harmonia com o que vem sendo defendido por Ferreira et al. (2011, p. 13), para os pesquisadores “" [...] o perfil empreendedor atual está mais dinâmico e complexo, exigindo características diferenciadas de acordo com o tipo de negócio, evidenciando a necessidade de constante adaptação".

Os recursos, no EI, podem ser desenvolvidos ao longo da trajetória da empresa pelo próprio empreendedor, a exemplo, da marca, instalações e conhecimento (Ribeiro \& Pimentel, 2009). O desafio está na identificação de fontes e maximização de recursos e, de posse deles, é possível elevar o padrão competitivo empresarial (Tondolo et al., 2008).

Podendo ser concebidas como um recurso, as redes de relacionamentos, no campo de negócios internacionais, possuem teoria própria, entretanto, a sua influência permeia todo processo de EI, por isso, podese dizer que é um fator determinante. As redes capazes de influenciar a internacionalização não se restringem às formadas na cadeia de suprimentos e com as mais diversas instituições. As redes de relacionamentos favorecem o acesso aos recursos (Ferreira et al., 2010) considerados estratégicos, tais como, conhecimento de mercado (Freitag Filho \& Amal, 2008; Tondolo et al., 2008), colaboram na identificação (Freitag Filho \& Amal, 2008; Rossi, 2008) e exploração de oportunidades (Ferreira et al., 2010; Mello et al., 2009), permitem o desenvolvimento de alianças estratégicas e outras formas de cooperação, favorece o desempenho (Freitag Filho \& Amal, 2008) e o sucesso da internacionalização (Ferreira et al., 2010; Freitag Filho \& Amal, 2008).

Em relação à propensão ao risco, o modo como o empreendedor os enfrenta para se internacionalizar está na essência do EI (Freitag Filho \& Amal, 2008; Mello et al., 2009; Rossi, 2008). A propensão ao risco é inevitável nessa abordagem teórica (Mello et al., 2009). O empreendedor não atua de forma irresponsável, porém, o fato de estar mais propenso a enfrentar determinadas situações para se internacionalizar, é o que o diferencia das demais pessoas (Mello et al., 2009).

O tamanho da firma é o tema de destaque que traz as pequenas e médias empresas como as mais evidenciadas nas pesquisas (Child \& Rodrigues, 2007; Freitag Filho \& Amal, 2008; Galimberti \& Fracasso, 2008; Galimberti, 2009; Mello et al., 2009), a exceção em duas publicações teórico-empíricas (Rossi, 2008; Tondolo et al., 2008) e nos ensaios teóricos (Dib \& Carneiro, 2006; Leite \& Moraes, 2011). Portanto, observa-se uma concentração de estudos de EI nas empresas de menor tamanho, típico de pesquisadores que estão na fase inicial do uso da literatura de EI.

O tempo de funcionamento da firma é mais um dos indicadores abordados. Na maior parte dos estudos, os autores defendem que o EI é um fenômeno das novas empresas (Ferreira et al., 2010; Ribeiro \& Pimentel, 2009), todavia, deve-se ampliar esse escopo, incluindo as que estão com mais tempo de mercado (Rossi, 2008) para ter alinhamento com o que se conhece sobre o assunto no âmbito internacional.

Esses temas foram apresentados separadamente por priorizar melhor visualização e entendimento. Porém, existe certo imbricamento entre determinados fatores do EI que ainda serão tratados nas seções seguintes. Mas, pode-se adiantar que as ações do empreendedor, a aprendizagem e conhecimento, a propensão ao risco, a identificação e exploração de oportunidades, as redes e os recursos estão inter- 
relacionados. Sobretudo, são elementos que reforçam o empreendedorismo internacional.

Essa explanação sobre o modo como o empreendedorismo internacional vem sendo pesquisado no país é fundamental tanto para esta pesquisa quanto para futuros estudos. Antes de tudo, desvenda em que estágio do conhecimento o assunto está, com isso, promove a identificação de lacunas teóricas a serem pesquisadas. Então, de posse do panorama das referências internacionais e nacionais de EI, apresenta-se a proposta do framework, na seção seguinte.

\section{DISCUSSÃO DA ANÁLISE BIBLIOGRÁFICA}

\subsection{Proposta do Framework de Empreendedorismo Internacional}

Há diversos desafios a serem ultrapassados para as empresas comercializarem produtos e serviços no mercado exterior. $\mathrm{Na}$ perspectiva do empreendedorismo internacional, existem dimensões ou características essenciais que promovem esse processo. Assim, propensão à adaptação, redes de relacionamentos, oportunidades internacionais, capacidade de inovar, atitude face ao risco e desenvolvimento de recursos competitivos constituem as dimensões do empreendedorismo internacional, que formam os eventos, aqui denominados PROCAD, identificadas a partir da análise crítica na literatura internacional e nacional (ver Figura 2).

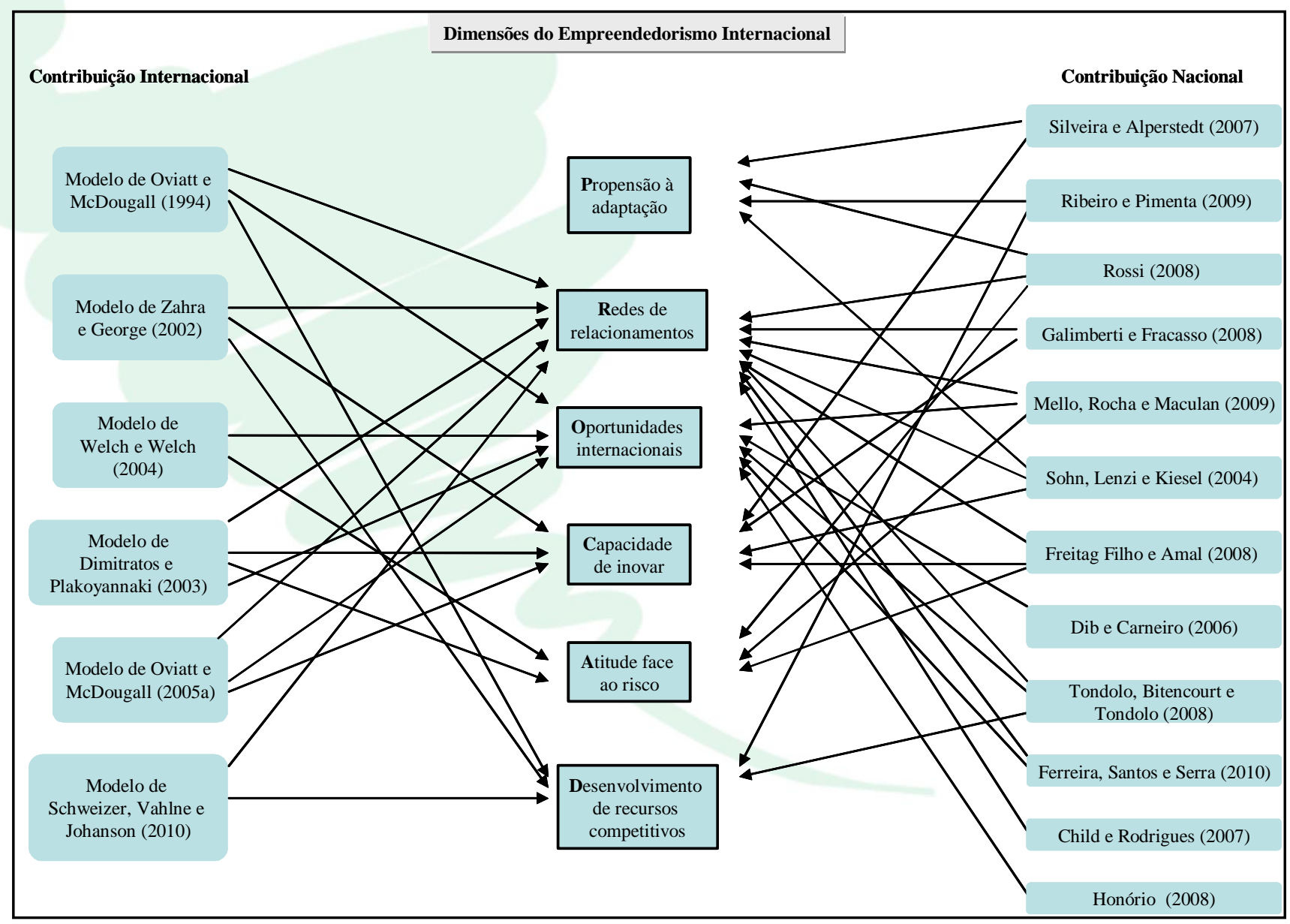

Figura 2 - Dimensões do Empreendedorismo Internacional Fonte: Elaborada pelos autores.

A figura 2 está sustentada pela revisão de literatura nas publicações de autores brasileiros, conforme já explanada na seção anterior, e estrangeiros. Os textos internacionais analisados foram selecionados pelo critério de representatividade e reconhecimento acadêmico, considerando dois fatores, 
quais sejam: aqueles que conceituam empreendedorismo internacional (Coombs et al., 2009; Dimitratos \& Plakoyiannaki, 2003; McDougall, 1989; Mtigwe, 2006; Oviatt \& McDougall, 1994; Oviatt \& McDougall, 2005a; Zahra \& George, 2002) e os que revelam os modelos teóricos de EI (Andersson, 2000; Dimitratos \& Plakoyiannaki, 2003; Oviatt \& McDougall, 1994; Oviatt \& McDougall, 2005a; Schweizer et al., 2010; Welch \& Welch, 2004; Zahra \& George, 2002).

As próximas seis seções concentram a discussão nas seis dimensões de EI. Salienta-se que a identificação do conjunto dessas dimensões está baseada na sua relevância e existência nas pesquisas desenvolvidas no Brasil, assim como, nas investigações realizadas por pesquisadores estrangeiros da área de empreendedorismo internacional.

\subsection{Propensão à Adaptação}

O empreendedor é um agente de mudança, responsável pela eliminação de barreiras comerciais e culturais ao desenvolver novas relações, quebrar paradigmas e gerar riquezas para a sociedade (Silveira \& Alperstedt, 2007). As ações empreendedoras embasam as mudanças que acontecem na empresa durante o processo de internacionalização (Freitag Filho, 2008), principalmente, devido ao fato do empreendedor estar atento às tendências e por ter visão de negócio (Ribeiro \& Pimentel, 2009). Nessa perspectiva, a capacidade de se adaptar ao ambiente é fundamental para a gestão estratégica (Tondolo et al., 2008).

A análise da interação dos gestores com o ambiente na busca do equilíbrio é objeto do trabalho clássico de Miles et al. (1978). Os autores denominam como Ciclo Adaptativo, o modelo que tem por base a reação frente aos problemas administrativos, de empreendedorismo e engenharia da empresa. Por esse debate, observa-se que a relação entre estratégia, estrutura e processo é fundamental para as pesquisas de adaptação e mudança organizacional (tal como já analisado por Miles et al., 1978). Na implementação das estratégias, os dirigentes consideram o tipo de estrutura podendo modificá-la para obter um ajuste adequado ao contexto (Chandler, 1998). Um exemplo disso acontece ao atender a demanda internacional, situação em que os empreendedores modificam a estrutura e os sistemas de controle (Coombs et al., 2009) com a inserção de departamentos, como o de marketing (Galimberti, 2009; Rossi, 2008) e modificações no setor de produção (Silveira \& Alperstedt, 2007).

Diante dessa discussão, percebe-se a existência de influências diversas no processo de adaptação das empresas internacionalizadas, interpretação que converge com a proposta de Etemad (2004). O sistema interativo com diferentes graus de complexidade e adaptabilidade, apresentado pelo autor, prevê interações entre as ações do empreendedor (ou/e sua equipe), a firma, o mercado e o ambiente internacional.

\subsection{Redes de Relacionamentos}

Redes, redes de relacionamentos, redes sociais e networks são palavras utilizadas como sinônimo neste ensaio teórico. Na perspectiva de Ferreira et al. (2010, p. 140): "As redes sociais são um veículo importante para as firmas com empreendedorismo internacional na identificação de oportunidades, mas também no acesso ao conhecimento, às inovações e aos recursos físicos". Por sua vez, Wright e Dana (2003) apresentam uma tipologia de networks: networks horizontais, networks verticais e networks trans-industriais.

A intolerância à incerteza tende a provocar dificuldades na tomada de decisões arriscadas. $\mathrm{Na}$ tentativa de minimizá-las, os empreendedores podem desenvolver redes para evitar a má-fé e adquirir confiança. Essa é uma das premissas do pensamento de Granovetter (1985). Além disso, o oportunismo pode ser enfraquecido com a internalização de algumas transações (Williamson, 1995) e por meio da integração vertical (Barney \& Hesterly, 2004). Entretanto, diferentemente de Williamson (1995) que compreende as transações unicamente pela perspectiva econômica, Granovetter (1985) insere a dimensão das relações sociais, sendo as redes de relacionamentos construídas como resultado do imbricamento entre os fatores social e econômico.

\subsection{Oportunidades Internacionais}

A identificação de oportunidades depende da relação entre o empreendedor e o contexto. Shane e Venkataraman (2000) explicam que esse processo depende da natureza das oportunidades (expectativa de retorno elevado) e das diferenças individuais (custo de oportunidade). Os empreendedores procuram oportunidades viáveis, que passem valor ao cliente para ter retornos financeiros elevados. Por outro lado, há custos de oportunidade. Os dirigentes deixam uma em prol de outra. Esse é um dos riscos enfrentados na atividade empreendedora, o qual depende da solução dos dilemas relativos às escolhas estratégicas (Lam \& White, 1999).

O comércio internacional é para Barney (2002), uma oportunidade de negócio. Muitas vezes, as imperfeições do mercado precisam ser identificadas pelo empreendedor (McDougall, 1989) para serem utilizadas em benefício da empresa. O papel do dirigente é essencial na internacionalização, conforme compartilhado por diferentes autores de negócios internacionais (Johanson \& Vahlne, 1977, 2009; Vernon, 1966). Ele pode explorar uma oportunidade por meio da transferência de eficiência (Bartlett \& Ghosbal, 1987), da aprendizagem e conhecimento (Bartlett \& Ghosbal, 1987; Vernon, 1966), da 
experiência (Johanson \& Vahlne, 1977, 2009), da mentalidade global (Nummela et al., 2004) e das networks (Johanson \& Vahlne, 2009). Assim, observase que a exploração de oportunidades depende das ações empreendedoras que viabilizam a internacionalização.

\subsection{Capacidade de Inovar}

Nos estudos desenvolvidos em 54 países pelo Global Entrepreneurship Monitor, a inovação é avaliada em termos dos produtos e serviços (Kelley et al., 2010). Hage (1999, p. 599) explica que a “[...] inovação organizacional tem sido consistentemente definida como a adoção de uma idéia ou um comportamento que seja novo para a organização [...]. A inovação pode ser um produto novo, um serviço novo, uma tecnologia nova ou uma prática administrativa nova". Esse é o conceito adotado nesta pesquisa, por não restringir a inovação ao bem ofertado ao cliente e está relacionado às práticas gerenciais inovadoras, o que se aproxima do fenômeno do empreendedorismo internacional.

No empreendedorismo internacional, a capacidade de inovar depende da criatividade do empreendedor. Zahra e George (2002) esclarecem que a criatividade pode ser de qualquer pessoa empreendedora que faz parte do time gerencial. Butler et al. (2010, p. 129) defendem que a "[...] criatividade é a característica do empreendedor que integra e otimiza seus processos cognitivos para produzir sucesso, é por isso que desempenha papel importante de apoio ao empreendedorismo internacional". Diante de sua importância, a inovação é compreendida como uma das dimensões do EI (Dimitratos \& Plakoyannaki, 2003).

$\mathrm{Na}$ literatura nacional, existem algumas semelhanças do que vem sendo discutido internacionalmente. A capacidade de inovar faz parte do EI (Freitag Filho \& Amal, 2008), sendo mais comum a sua presença nas empresas do setor de tecnologia - software (Galimberti \& Fracasso, 2008; Sohn et al., 2004). As ideias inovadoras são fundamentais, assim como, a criatividade (Silveira \& Alperstedt, 2007).

\subsection{Atitude Face ao Risco}

A propensão ao risco é o “[...] traço da personalidade do tomador de decisão que influencia o comportamento de tomada de risco, mas não o determina" (Grichnik, 2008, p. 25). Nesse caso, os traços da personalidade do empreendedor e a sua percepção apresentam-se como propulsores da tomada de risco.

O risco abordado neste estudo é aquele enfrentado pelos empreendedores para internacionalizar a empresa que depende da disposição de comprometer recursos (Lumpkin \& Dess, 1996). Existem diferentes fontes de risco no processo de internacionalização. A exploração de oportunidades por si é arriscada (Johanson \& Vahlne, 2009), os gestores podem tomar decisões que sejam negativas à empresa. Outra fonte de riscos é a utilização dos modos de entrada (Sharma \& Erramili, 2004). Mas para reduzir tais riscos, Melin (1992), Lam e White (1999) argumentam que a internacionalização gradual iniciada com a exportação, favorece a aprendizagem.

A propensão ao risco permite a realização de uma internacionalização agressiva (Mello et al., 2009). Esse é um pensamento compartilhado por Mtigwe (2006) e Dimitratos e Plakoyannaki (2003), que apresentam a atitude ao risco internacional como um dos elementos do EI.

\subsection{Desenvolvimento de Recursos Competitivos}

$\mathrm{Na}$ internacionalização os recursos competitivos são essenciais. A sua escassez pode inviabilizar a consolidação das negociações com os compradores estrangeiros (Keupp \& Gassmann, 2009). A estrutura física, os recursos financeiros, a localização, a obtenção de incentivos do país anfitrião e os recursos humanos formam o pool de recursos tangíveis. Nesse caso, eles estão relacionados à empresa e aos gestores/empreendedores. Essas vantagens de propriedade contribuem nas ações de produzir, vender, comercializar, e transferir bens (Sharma \& Erramilli, 2004).

Há uma semelhança entre os modelos de EI apresentados por Oviatt e McDougall (1994) e Zahra e George (2002), nos dois os autores enfatizam o poder dos recursos na conquista da vantagem competitiva. Tal entendimento também é compartilhado por Mtigwe (2006) que apresenta a vantagem de recursos como uma referência na constituição do EI.

\subsection{Framework de Empreendedorismo Internacional}

Nesta seção é exposta uma proposta preliminar baseada na revisão de literatura. Dessa forma, optou-se por construir um framework. A Figura 3 apresenta o framework de empreendedorismo internacional expressado pelo conjunto de eventos PROCAD que ocorrem ao longo da trajetória da internacionalização. As seis dimensões constituem a dinâmica de cada evento, as quais recebem influências do ambiente externo. Todavia, cabe o pressuposto de que nem sempre as seis dimensões estarão presentes em todos os eventos, o que poderá (ou não) ser constatado após a realização de pesquisas empíricas.

A Figura 3 é resultado da discussão teórica desenvolvida e se fundamenta na intersecção dos construtos evidenciados na literatura. 


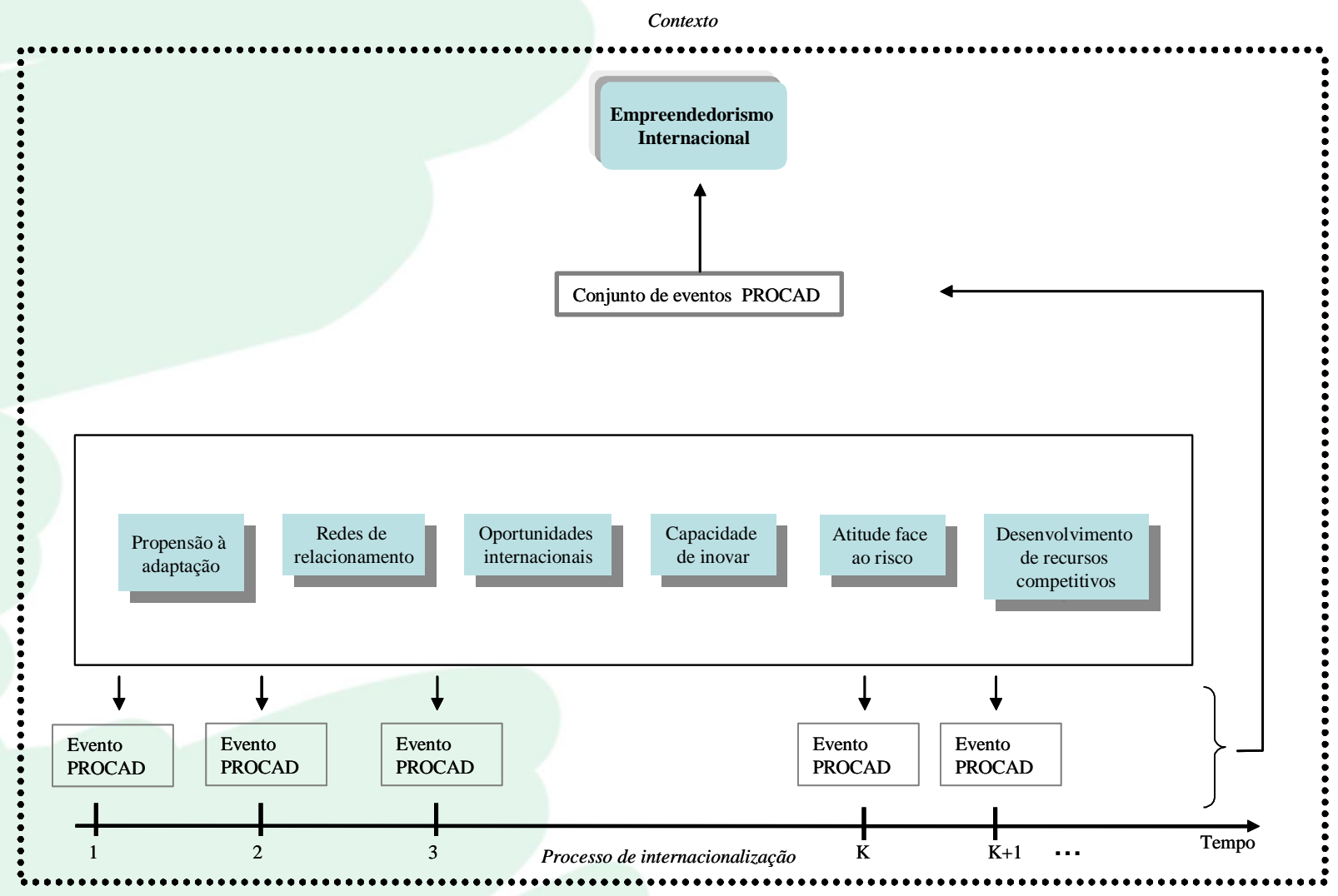

Figura 3 - Proposição do framework de Empreendedorismo Internacional Fonte: Elaborada pelos autores.

Em relação ao ambiente externo, por se tratar de pesquisa sobre empreendedorismo internacional como processo, o contexto é determinante (Dimitratos \& Plakoyiannaki, 2003). Autores da área, como Mtigwe (2006), também argumentam esse pressuposto, principalmente, por reconhecer a força do ambiente externo nas atitudes do empreendedor internacional. As decisões dos empreendedores são comprometidas com os acontecimentos do ambiente externo e as capacidades empreendedoras podem ser fortalecidas de acordo com as condições sociais nas quais a empresa está inserida (Acs et al., 2003).

Nos países emergentes, o EI é menos sofisticado quando comparado com a realidade dos países desenvolvidos (Kiss et al., 2012). A internacionalização de empresas localizadas naquele contexto tende a acontecer com o menor uso de tecnologia industrial intensiva e com menos custo de desenvolvimento de produto.

O contexto que engloba a esfera internacional e nacional influencia o EI significativamente. Nele estão fatores como a economia, a demografia, a tecnologia, a cultura, o ambiente político-legal, os concorrentes, dentre outros. Esse contexto pode determinar o processo de internacionalização de uma empresa, assim como, a dinâmica das seis dimensões que subsidiam o empreendedorismo internacional.
A observância da interação das forças do ambiente externo é apontada por Bartlett e Ghoshal (1987) como sendo essencial na transição para a transnacionalidade. As capacidades estratégicas ganham status e atenção para favorecer o intercâmbio entre a realidade externa e a organização. Com essa abordagem, as multinacionais são motivadas a executar a transferência eficiente de inovações domésticas e expertise nas operações em outros países, em diferentes contextos.

A presença das seis dimensões no decorrer da internacionalização forma cada evento PROCAD, o conjunto desses eventos origina o empreendedorismo internacional. Entende-se que para se ter o fenômeno do EI, é necessária a continuidade da implementação de eventos PROCAD ao longo do processo de internacionalização.

Esse entendimento partiu do pressuposto de ser a internacionalização um processo. Van de Ven (1992) sugere três diferentes definições, destacando-se entre elas o conceito de processo como uma sequência de eventos, provocados por ações individuais $\mathrm{e}$ organizacionais. Por meio desse conceito, foi possível elaborar a proposta de evento PROCAD. Sendo assim, formula-se a seguinte proposição: 
- O empreendedorismo internacional ocorre quando existe a presença contínua de eventos PROCAD no processo de internacionalização da empresa.

\section{CONSIDERAÇÕES FINAIS}

O framework de empreendedorismo internacional proposto contempla uma abordagem processual e contextualizada, formado por eventos marcantes e dimensões típicas. Não obstante, o framework apresentado indique a existência de eventos constituídos por seis dimensões (propensão à adaptação, redes de relacionamentos, oportunidades internacionais, capacidade de inovar, atitude face ao risco e desenvolvimento de recursos competitivos), acredita-se que ao longo do processo de internacionalização exista uma variação na incidência desse conjunto de elementos, aspecto do modelo que deve ser aprofundado em pesquisas teórico-empíricas futuras.

Os contextos nacional e internacional podem ser determinantes no sentido de proporcionar condições para as ações empreendedoras. Os fatores do ambiente não apenas afetam o processo de internacionalização como também a expressividade de cada uma das dimensões típicas de EI. Por exemplo, as oportunidades internacionais não somente dependem da visão do empreendedor mas também do que o ambiente proporciona. Desse modo, o framework exposto contempla os principais aspectos dos modelos existentes e extrapola o conhecimento da área ao apontar que as dimensões do EI estão presentes nos eventos marcantes do processo de internacionalização.

De posse dos dados empíricos, o modelo será aprimorado conforme a realidade contemporânea. No momento, o que se apresenta é uma proposição baseada nas teorias existentes, sobre a forma de ensaio.

Outro fator de destaque está na indicação de se realizar, na primeira etapa de pesquisas futuras, a abordagem qualitativa, para apenas em seguida, adotar a perspectiva quantitativa, na mesma investigação ou num segundo momento, em outra pesquisa. Esse fato trata-se de uma limitação do modelo por ter como princípio a necessidade de desenvolver o exame das dimensões considerando o processo de internacionalização de forma contextualizada. Além disso, como implicação para investigações futuras, observa-se que os fatores de processo e contextuais favorecem a aplicação do framework de EI.

\section{REFERÊNCIAS}

Acs, Z., Dana, L., Jones, M. V. (2003). Toward new horizons: the internationalisation of entrepreneurship. Journal of International Entrepreneurship, 1(1), 5-12.

Barney, J. B. (2002). Gaining and Sustaining Competitive Advantage. 3. ed. New Jersey: Prentice-Hall.

Barney, J. B., Hesterly, W. (2004). Economia das organizações: entendendo a relação entre as organizações e a análise econômica. In S. CLEGG, C. HARDY e D. NORD D. (Orgs.), Handbook de estudos organizacionais. Volume 3. São Paulo: Atlas, 131-179.

Bartlett, C. A., Ghoshal, S. (1987). Managing across Borders: New Strategic Requirements. Sloan Management Review, 28(4), 7-17, Summer.

Butler, J. E., Doktor, R., Lins, F. A. (2010). Linking international entrepreneurship to uncertainty, opportunity discovery, and cognition. Journal of International Entrepreneurship, 8(2), 121-134.

Chandler, A. (1998). Introdução a strategy and struture. In A. CHANDLER, Ensaios para uma teoria histórica da grande empresa. Rio de Janeiro: Fundação Getúlio Vargas, pp. 121-140.

Child, J., Rodrigues, S. B. (2007). The Role of Trust in International Entrepreneurship. In: XXXI Encontro da ANPAD, Rio de Janeiro, 2007. Anais... Rio de Janeiro (Brasil): EnAnpad.

Coombs, J. E., Sadrieh, F., Annavarjula, M. (2009). Two decades of international entrepreneurship research: what have we learned - where do we go from here? International Journal of Entrepreneurship, 13(1), 23-64.

Dana, L. (2004). Handbook of research on international entrepreneurship. Cheltenham, UK: Edward Elgar.

Dib, L. A., Carneiro, J. (2006). Avaliação Comparativa do Escopo Descritivo e Explanatório dos Principais Modelos de Internacionalização de Empresas. In: XXX Encontro da ANPAD, Salvador, 2006. Anais... Salvador: EnAnpad.

Dib, L. A. (2008). O processo de internacionalização de pequenas e médias empresas e o fenômeno born global: estudo do setor de software no Brasil. 2008. 352f. Tese (Doutorado em Administração) Programa de Pós-Graduação em Administração, Universidade Federal do Rio de Janeiro, Rio de Janeiro.

Dimitratos, P., Plakoyiannaki, E. (2003). Theoretical foundations of international entrepreneurial culture. 
Journal of International entrepreneurship, 1(2), 187-215.

Etemad, H. (2004). International entrepreneurship as a dynamic adaptive system: towards a grounded theory. Journal of International Entrepreneurship, 2(1), 15-59.

Ferreira, L. F. F. et al. (2011). «Desde os Primórdios até hoje em dia: Será que o Empreendedor ainda faz o que Schumpeter dizia? Evolução das Características Empreendedoras de 1983 a 2010». In: XXXV Encontro da ANPAD, Rio de Janeiro, 2011. Anais... Rio de Janeiro: EnAnpad.

Ferreira, M. P., Santos, J. C., Serra, F. A. R. (2010). The international entrepreneurial firms' social networks. Cadernos EBAPE.BR, 8(1), 133-145.

Filion, L. J. (1999). Empreendedorismo: empreendedores e proprietários-gerentes de pequenos negócios. Revista de Administração, São Paulo, 34(2), 5-28, Abr/Jun.

Freitag Filho, A. R., Amal, M. (2008). Estratégias e Determinantes da Internacionalização de Pequenas e Médias Empresas (PMEs): Abordagem da Teoria de Redes de Relacionamento e Empreendedorismo. In: XXXII Encontro da ANPAD, Rio de Janeiro, 2008. Anais... Rio de Janeiro: EnAnpad.

Freitag Filho, A. R. (2008). Estratégias e determinantes da internacionalização de pequenas e médias empresas catarinenses: abordagem da teoria de redes de relacionamento e empreendedorismo. 2008. 129 f. Dissertação (Mestrado em Administração) - Programa de Pós-Graduação em Administração, Universidade Regional de Blumenau, Blumenal.

Galimberti, M. F., Fracasso, E. M. (2008). Inovação e Empreendedorismo nas Teorias de Internacionalização de Empresas Aplicáveis às Pequenas e Médias Empresas de Software e Serviços Correlatos. In: XXV Simpósio de Gestão da Inovação Tecnológica da ANPAD, Brasília, 2008. Anais... Brasília: Simpósio de Gestão da Inovação Tecnológica.

Galimberti, M. F. (2009). Fatores de sucesso na internacionalização ativa de pequenas e médias empresas de software: estudo de casos do Brasil e da França. 2009. 213f. Tese (Doutorado em Administração) - Programa de Pós-Graduação em Administração, Universidade Federal do Rio Grande do Sul, Porto Alegre.
Granovetter, M. (1985). Economic action and social structure: the problem of embeddedness. American Journal of Sociology, 91(3), 481-519.

Grichnik, D. (2008). Risk choices in new ventures decisions: experimental evidence from Germany and the United States. Journal of International Entrepreneurship, 6(1), 22-47.

Hage, J. T. (1999). Organizational innovation and organizational change. Annual Review of Sociology, 25, 597-622.

Honório, L. C. (2008). A internacionalização de empresas brasileiras em uma perspectiva motivacional. Revista de Administração Mackenzie, 9(2), 128-151.

Johanson, J., Vahlne, J. (1977). The internationalization process of the firm: a model of knowledge development and increasing foreign markets commitments. Journal of International Business Studies, 23-32, Spring.

Johanson, J., Vahlne, J. (2009). The Uppsala Internationalization Process Model Revisited: from liability of foreignness to liability of outsidership. Journal of International Business Studies, 40, 14111431.

Jones, M. V., Coviello, N., Tang, Y. K. (2011). International entrepreneurship research (19892009): a domain ontology and thematic analysis. Journal of Business Venturing, 26(1), 632-659.

Kelley, D. J., Bosma, N., Amorós, J. E. (2010). Global Entrepreneurship Monitor: 2010 global report. Babson e Universidad del Desarrollo.

Keupp; M. M., Gassmann, O. (2009). The past and the future of international entrepreneurship: a review and suggestions for developing the field. Journal of Management, 35(3), June.

Kiss, A. N., Danis, W. M., Cavusgil, S. T. (2012). International entrepreneurship research in emerging economies: a critical review and research agenda. Journal of Business Venturing, 27(Issue 2), March 266-290.

Lam, L. W., White, L. P. (1999). An adaptative choice model of the internationalization process. International Journal of Organizational Analysis, 7(2), April, 105-134.

Lumpkin, G. T., Dess, G. G. (1996). Clarifying the entrepreneurial orientation construct and linking it to performance. Academy of Management, 21(1), Jan. 135-172. 
Mcdougall, P. P. (1989). International versus domestic entrepreneurship: new venture strategic behavior and industry structure. Journal of Business Venturing, 4(6), 387-400.

Melin, L. (1992). Internationalization as a strategy process. Strategic Management Journal, 13, 99118.

Mello, R. C. de, Rocha, A. da, Maculan, A.-M. (2009). A Trajetória Internacional das Pequenas Empresas: É Possível Conciliar as Teorias Comportamentais?. In: IV ENCONTRO DE ESTUDOS EM ESTRATÈGIA, Recife, 2009. Anais... Recife: 3Es.

Mello, R. C. de. (2009). O processo de internacionalização de empresas brasileiras de software: reconciliando diferentes correntes teóricas. Tese (Doutorado em Engenharia de Produção) - Universidade Federal do Rio de Janeiro.

Miles, R. E., Snow, C. C., Meyer, A. D., Coleman, H. J. (1978). Organizational strategy, structure, and process. Academy of Management Review, 3(3), Jul. 546-562.

Morrow, J. F. (1988). International entrepreneurship: a new growth opportunity. New Management, 5(3), 59-60.

Mtigwe, B. (2006). Theoretical milestones in international business: the journey to international entrepreneurship theory. Journal of International Entrepreneurship, 4(1), 5-25.

Oviatt, B. M., McDougall, P. P. (1994). Toward a theory of international new ventures. Journal of International Business Studies, 25(1), 45-64.

Oviatt, B. M., McDougall, P. P. (2005a). Defining international entrepreneurship and modeling the speed of internationalization. Entrepreneurship Theory \& Practice, 29(5), 537-553.

Oviatt, B. M., McDougall, P. P. (2005b). Toward a theory of international new ventures. Journal of International Business Studies, 36(1), 29-41.

Oviatt, B. M., McDougall, P. P. (2005c). The internationalization of entrepreneurship. Journal of International Business Studies, 36(1), 2-8.

Ribeiro, F. C. F., Pimentel, J. E. A. (2009). Empresas Born Globals Brasileiras: a Influência do Perfil do Empreendedor e da Localização Geográfica. In: XXXIII Encontro da ANPAD, São Paulo, 2009. Anais... São Paulo: EnAnpad.
Rossi, D. L. (2008). Dimensões do empreendedorismo internacional na internacionalização de uma empresa mineira do setor alimentício. Dissertação de Mestrado Acadêmico em Administração, Programa de Pós-Graduação em Administração. Faculdade Novos Horizontes, Belo Horizonte, $114 \mathrm{f}$.

Schweizer, R., Vahlne, J.-E., Johanson, J. (2010). Internationalization as an entrepreneurial process. Journal of International Entrepreneurship, 8(4), 343-370.

Shane, S., Venkataraman, S. (2000). The promise of entrepreneurship as a field of research. Academy of Management Review, 25(1), 217-226, jan.

Sharma, V. M., Erramilli, M. K. (2004). Resourcebased explanation of entry mode choice. Journal of Marketing Theory and Practice, 12(1), Winter, 118.

Silveira, P. A., Alperstedt, G. D. (2007). O Processo de Internacionalização de uma Empresa de Pequeno Porte do Setor Moveleiro do Oeste de Santa Catarina sob a Ótica do Empreendedor. In: III ENCONTRO DE ESTUDOS EM ESTRATÉGIA, São Paulo, 2007. Anais... São Paulo: 3Es.

Sohn, A. P. L., Lenzi, F. C., Kiezel, M. D. (2004). A Presença do Espírito Empreendedor no Processo de Formulação de Estratégias de Internacionalização da Datasul. In: XXVIII Encontro da ANPAD, Curitiba, 2004. Anais... Curitiba: EnAnpad.

Tondolo, V. A. G., Bitencourt, C. C., Tondolo, R. da R. P. (2008). Implementação de Estratégia Empreendedora Internacional no Setor de Vinhos: O Caso da Vinícola Miolo. In: XXXII Encontro da ANPAD, Rio de Janeiro, 2008. Anais... Rio de Janeiro: EnAnpad.

Van de Ven, A. H. (1992). Suggestions for studing stratey process: a research note. Strategic Management Journal, 13, 169-188.

Vernon, R. (1966). International investment and international trade in the product cycle. Quarterly Journal of Economics, 191-207, 1966.

Welch, C., Welch, L. S. (2004). Broadening the concept of international entrepreneurship: internationalization, networks and politics. Journal of International Entrepreneurship, 2(3), 217-237, Sep.

Williamson, O. E. (1995). Transaction cost economics and organization theory. In O. E. Williamson (Ed.). Organization theory from Chester Barnard to the present and beyond. Expanded Edition. New York: Oxford University Press, 207-256. 
Wright, R. W., Dana, L.-P. (2003). Changing paradigms of international entrepreneurship strategy. Journal of International Entrepreneurship, 1(1), 135-152.
Zahra, S. A., George, G. (2002). International entrepreneurship: the current status of field and future research agenda. In M. A. HITT, R. D. IRELAND, D. L. SEXTON e S. M. AMP (Eds.), Strategic entrepreneurship, creating a new mindset. Oxford, UK: Blackwell, 255-288. 